\title{
A Raman spectroscopic study of bulk and surface ageing phenomena in polyethylene
}

\author{
X. S. Liu ${ }^{1}$, A. S.Vaughan ${ }^{2}$ and G. Chen ${ }^{2}$ \\ ${ }^{1}$ Xian Jiaotong University, China \\ ${ }^{2}$ University of Southampton, Southampton, UK
}

\begin{abstract}
Low density polyethylene (LDPE) films were subjected to a range of electrical stresses. Analysis of samples aged in the bulk by Raman microprobe spectroscopy indicated that changes in the spectra are only observed when samples are subjected to high electrical stresses for long periods of time. Even then, no specific chemical effects were detected; degradation only manifested itself in the form of mild fluorescence. Corona-induced surface reactions were also examined. Although the surface texture was found to change radically, subsequent Raman analysis revealed few chemical changes in the polymer, implying that erosion involves fragmentation of the LDPE and the formation of volatile by-products. No residues were detected on the samples. In addition to the LDPE itself, the corona electrode was also analyzed. This revealed the presence of extensive deposits with the fingerprint of $\mathrm{sp}^{2}$ hybridized carbon. The significance of these results are discussed with respect to high field ageing processes, particularly, the development of electrical trees.
\end{abstract}

\section{Introduction}

High voltage insulation materials are essential parts of all modern generation and transmission systems and their reliability is therefore critical to system performance. In recent years, polymeric insulation has largely replaced more traditional materials due to its low cost and excellent electrical properties. However, properties deteriorate over time, when the insulation is subjected to high electric stresses, and this topic has, therefore, attracted much study [1]. For example, chemical changes have been examined by spectroscopic means, including fluorescence spectroscopy [2] and, of particular relevance to this work, the Raman microprobe technique [3]. Unlike many other approaches, this has the potential advantage of high spatial resolution. An initial analysis suggested a theoretical lateral resolution of the order of $\sim 1 \mu \mathrm{m}$ and a resolution along the axis of the optical beam of $\sim 2 \mu \mathrm{m}$ [4]. For this reason, the approach was used to study local ageing effects in LDPE [3]. This work suggested that dramatic changes in the Raman response occur rapidly when the insulation experiences a nominal electric stress of $60 \mathrm{kV} / \mathrm{mm}$. Specifically, massive fluorescence was seen after just $1 \mathrm{~min}$, followed by the development of peaks around $1500 \mathrm{~cm}^{-1}$, one of which was attributed to the formation of $\mathrm{C}=\mathrm{C}$ bonds. This suggestion is in line with earlier proposals based upon experiments that, similarly, used a pin/plane electrode geometry $[5,6]$.

\section{Experimental}

Sheets of additive-free LDPE, $\sim 100 \mu \mathrm{m}$ in thickness, were used in all our experiments. To examine volume effects, samples were aged using a parallel electrode geometry, to provide a uniform field. For this, the LDPE was sandwiched between a brass high voltage (HV) electrode and a ground electrode formed from semiconducting polymer. The HV electrode was profiled, to reduce stress enhancement at its edges, and was cast in epoxy resin, both to prevent surface flashover and to attach it firmly to the sample. This was connected, via a breakdown protection system, to a $15 \mathrm{kV}$ (rms) AC power supply. Samples were exposed to electric fields of $50-100 \mathrm{kV} / \mathrm{mm}$ at room temperature. To study surface effects, film samples $50 \mathrm{~mm}$ in diameter, were placed on a grounded aluminium base-plate beneath an HV tungsten needle electrode (radius $\sim 3 \mu \mathrm{m}$ ). The interelectrode separation could be varied in the range 1.5$4.0 \mathrm{~mm}$ such that a corona discharge was formed above the sample. In all the experiments discussed here, an AC voltage of $5 \mathrm{kV}$ (rms) was used. Analysis of the above samples was performed using a Renishaw RM1000 confocal Raman microprobe system, including a Renishaw NIR 780TF diode laser (wavelength $780 \mathrm{~nm}$ ) with a maximum output power of $25 \mathrm{~mW}$.

\section{Results}

\section{Bulk ageing}

Initially, 12 samples were aged at each of the following applied fields: 50, 60, 80 and $100 \mathrm{kV} / \mathrm{mm}$; each test continued until 10 had failed. Examination of failed samples suggested that ageing should take place throughout the region beneath the HV electrode, since very few breakdowns occurred at their edges. To investigate associated chemical changes, Raman spectra were then acquired from those samples that had not failed. From Fig.1, which contains typical data, it is evident that none of these specimens appears significantly different from unaged LDPE. A further batch of 21 samples was therefore stressed at $50 \mathrm{kV} / \mathrm{mm}$ 


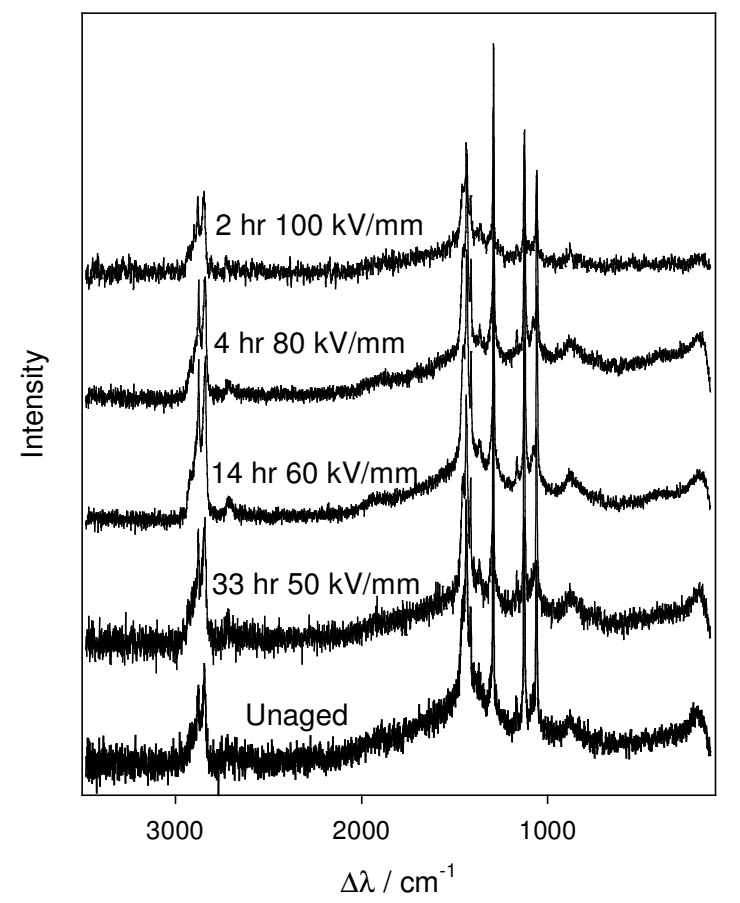

Figure 1: Effect of bulk ageing conditions on the Raman spectrum of LDPE.

until just one sample remained in tact, after 45 hrs. Figure 2 shows representative Raman spectra acquired from samples that had survived different ageing times at this applied field. Although no additional peaks are evident, the background Raman signal from the sample that had been aged for $45 \mathrm{hrs}$ at $50 \mathrm{kV} / \mathrm{mm}$ does rise progressively with decreasing wavenumber. This is associated with fluorescence and is commonly encountered in degraded samples [3,7]. However, since fluorescence can be associated with many transitions, it is not possible to extract chemical data from this; it merely provides a crude indication of degradation.

\section{Surface ageing}

Figure 3 shows the typical surface appearance of a corona-aged film. In this, it is difficult to make out clear structural detail, due to the extreme surface roughness; the texture shown is best thought of as raised regions (eg at A), separated by large eroded "valleys" (eg at B out of focus). Comparison of many samples indicates that, while the severity of erosion generally increases with ageing time, field etc, the local surface texture can also vary considerably. Raman data acquired from such an eroded surface are shown in Fig.4. The lower trace is typical of the Raman response of the matrix. Although this was acquired in confocal mode, and therefore

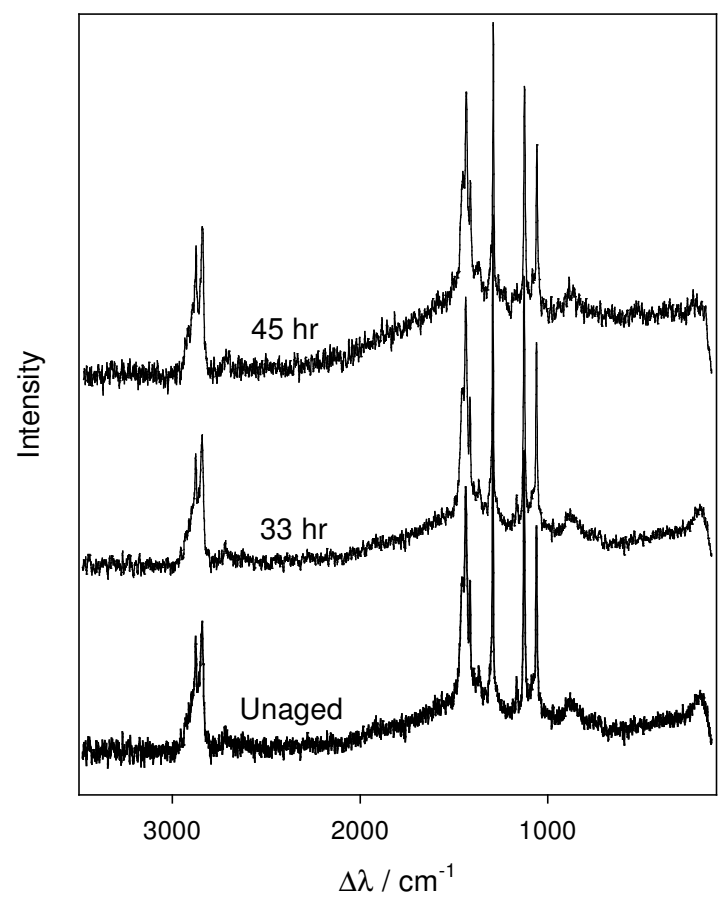

Figure 2: Effect of ageing time at $50 \mathrm{kV} / \mathrm{mm}$ on the Raman spectrum of LDPE. Some fluorescence is visible after $45 \mathrm{hr}$.

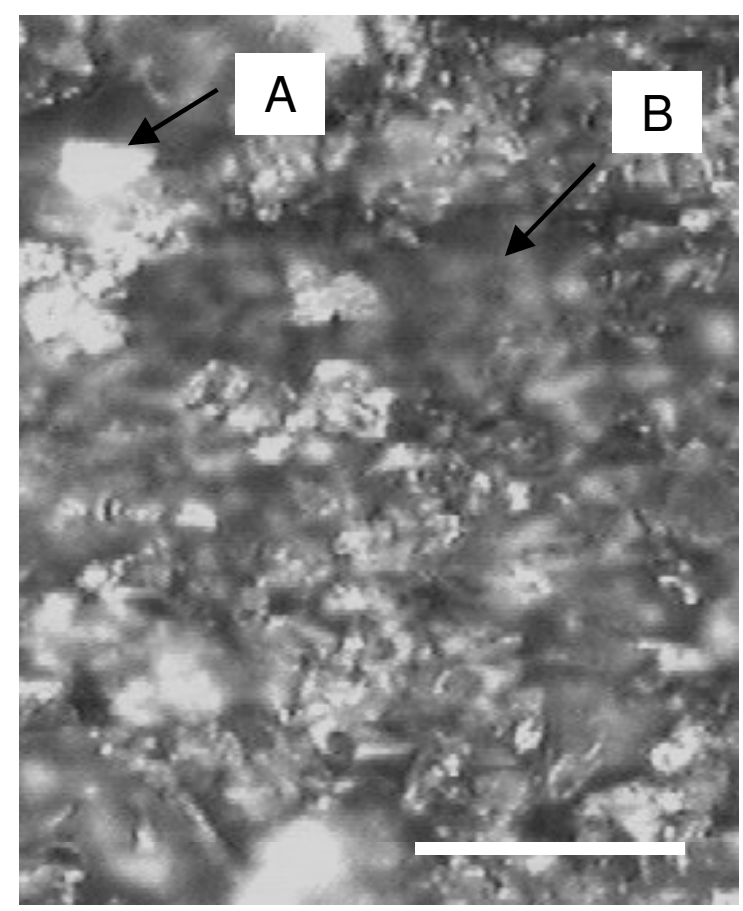

Figure 3. Optical micrograph showing the texture of a corona-aged LDPE film: $72 \mathrm{hr} ; 2 \mathrm{~mm}$ electrode spacing. Scale bar $-40 \mu \mathrm{m}$. 


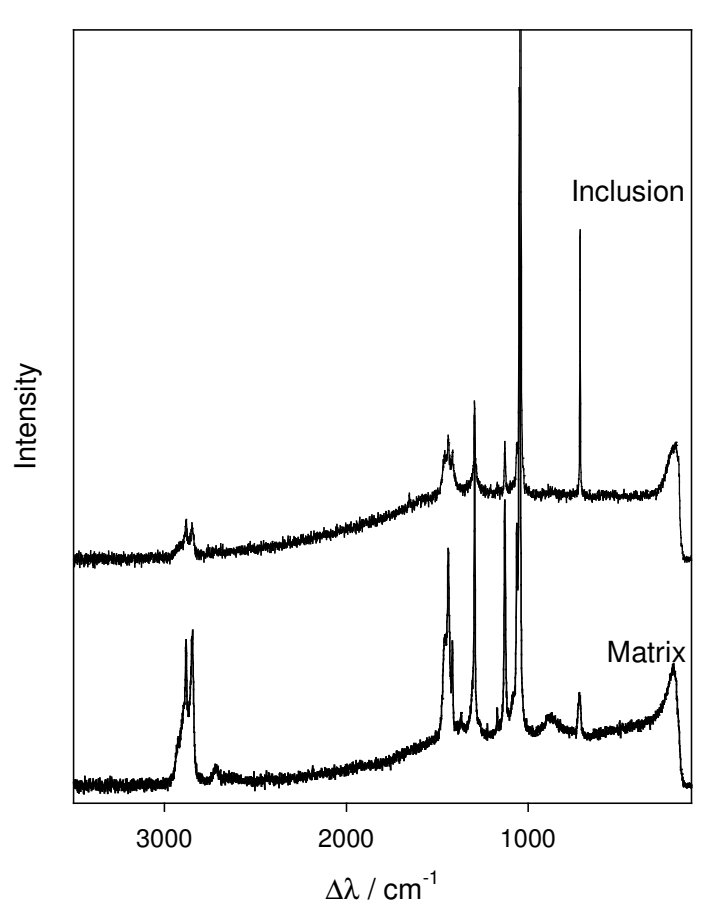

Figure 4: Typical Raman spectra acquired from a corona, surfaceaged sample of LDPE.

should be surface specific [3], such spectra appear very similar to unaged LDPE (see Fig.1). The second spectrum in Fig.4 was obtained from a raised region containing a type of inclusion that was commonly observed. Indeed, A in Fig.3, which appears highly reflective, corresponds to just such a feature. The visual appearance of this, together with Raman peaks at 713 and $1042 \mathrm{~cm}^{-1}$ [7] strongly suggest that this is an inorganic artefact. As such, the most likely explanation for its presence is dust particles that are electrostatically attracted to the surface and, subsequently, serve to protect the underlying polymer from further erosion. From Figs. 3 and 4 it would appear that the corona generates species that erode the polymer, but without inducing major changes in the remnant film. The common decomposition mode of polyethylene is through random chain scission to give volatile molecular fragments [8].

\section{The HV electrode}

Following a sequence of surface ageing experiments, a solid deposit was observed on the HV needle electrode. This is shown in Fig.5, together with typical Raman results in Fig.6. From Fig.6, it is clear that this can be characterized by three distinct features, the relative proportions of which vary from location-to-location.

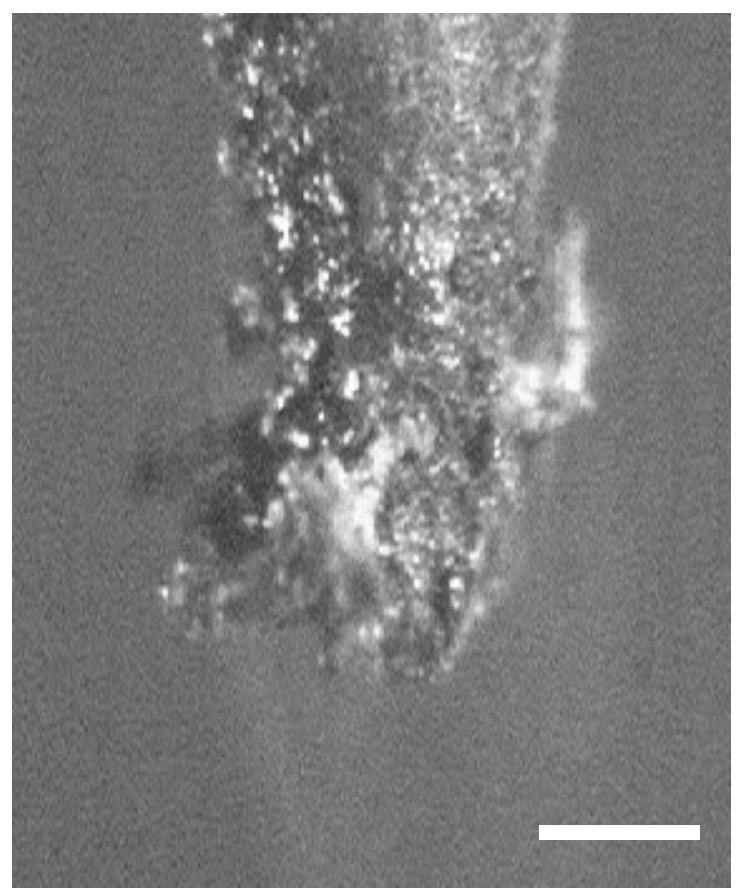

Figure 5: Optical micrograph showing accumulated deposits on the HV needle electrode. Scale bar $-200 \mu \mathrm{m}$.

These are: strong fluorescence; strong peaks centred at $\sim 1380$ and $\sim 1585 \mathrm{~cm}^{-1}$; a group of peaks in the range $250-650 \mathrm{~cm}^{-1}[9]$. The small peaks at $\sim 710$ and $\sim 1050 \mathrm{~cm}^{-1}$ in the upper trace are probably artefacts.

\section{Discussion}

Published results on bulk ageing [3] indicate that Raman peaks around $1500 \mathrm{~cm}^{-1}$, which have been attributed to the formation of $\mathrm{C}=\mathrm{C}$ bonds, are seen in LDPE after ageing with a pin/plane electrode system for just $11 \mathrm{~min}$ at a nominal electric stress of $60 \mathrm{kV} / \mathrm{mm}$. In contrast, our data, which were obtained from many samples, show few changes, even though the applied fields ranged from 50 to $100 \mathrm{kV} / \mathrm{mm}$. In our opinion, the explanation for this difference lies in the different electrode arrangements used in this study and in [3]. In the latter case, the field experienced by the material in the vicinity of the needle electrode will be much higher than the quoted nominal value, which is based simply on the applied voltage and electrode separation. Indeed, it is likely to be even higher than the $100 \mathrm{kV} / \mathrm{mm}$ used in the present study. However, from our results, local fields of this magnitude are likely to exceed the breakdown strength of the polymer and, then, the bulk degradation process will be very different from that considered here. Indeed, our studies of treeing processes in polyethylene [10] have demonstrated that internal 


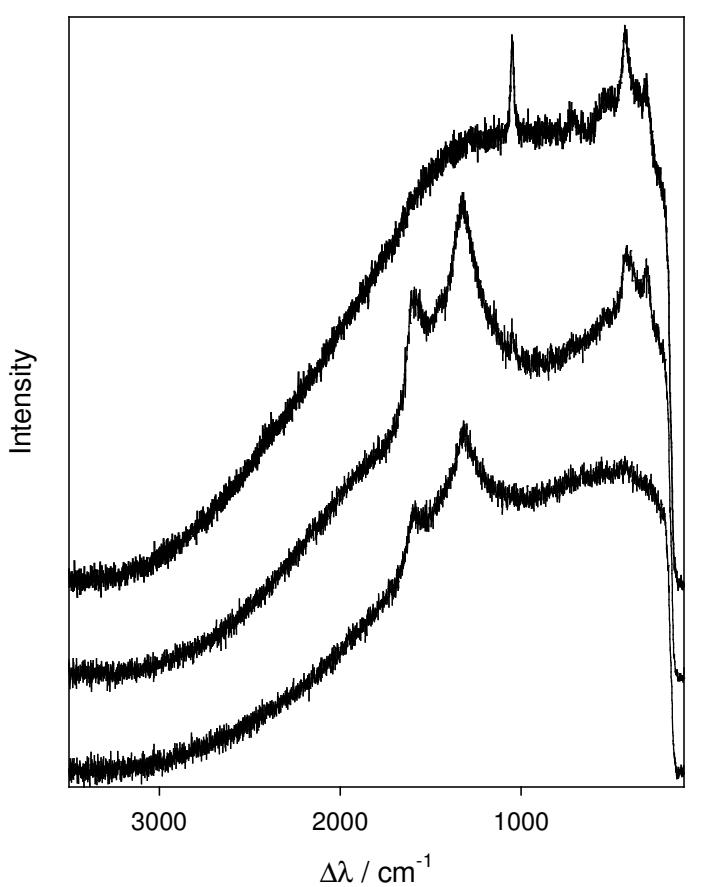

Figure 6: Typical Raman spectra acquired from the residues formed on the corona electrode.

discharges can generate precisely the same Raman fingerprints as those previously associated with ageing through electromechanical chain scission [3]. We believe this to be the origin of the discrepancy. However, of more general importance are the surface results which provide a direct mechanism by which $\mathrm{sp}^{2}$ hybridized carbon may be formed. While the signature of $\mathrm{sp}^{2}$ hybridized carbon within a certain class of electrical trees is clear, the processes that lead to it are not. We suggest that this material, which largely determines the electrical characteristics of electrical trees, is generated as follows. Partial discharge activity occurs within the tree, such that the solid polymer decomposes into volatile molecular fragments. When these are able to diffuse rapidly away and experience no further electrical activity, the resultant tree is made up of an array of hollow tubules. Conversely, where discharges occur within an atmosphere rich in organic molecules, as in the case of the needle electrode above, further reactions occur to form a solid graphitic residue. Whilst this suggestion is speculative at present, it is consistent with a range of observations.

\section{Conclusions}

The Raman response of LDPE has been examined under a number of different electrical ageing conditions. The absence of specific chemical changes, following bulk ageing with a parallel electrode geometry, is in marked contrast to published work. Surface ageing by exposure to a corona results in erosion of the polymer, to form volatile molecular fragments. No related changes in surface chemistry were detected. Raman spectra involving functional groups similar to those previously attributed to either mechanical backbone scission or injected carriers have been detected, where volatile organic decomposition products underwent subsequent reactions in the corona region. The similarity of these compounds to those detected within certain types of electrical trees suggests that a common process of formation occurs in both situations; discharge activity within an atmosphere rich in organic molecules formed by decomposition of the polymer.

\section{References}

[1] L. A. Dissado and J. C. Fothergill, Electrical Degradation and Breakdown in Polymers, edited by G. C. Stevens, London, UK: Peter Perigrinus, 1992.

[2] V. Griseri, L. A. Dissado, J. C. Fothergill, C. Laurent and G. Teyssèdre, "Photoluminescence, recombination induced luminescence and electroluminescence in epoxy resin", J. Phys. D: Appl. Phys., vol.34, pp. 2534-2540, Aug. 2001.

[3] P. Sayers, T. J. Lewis, J. P. Llewellyn and C. L. Griffiths, "Investigation of the structural changes in LDPE and XLPE induced by high electrical stress," in Dielectric Materials, Measurements and Applications, no. 473 in IEE Conference Proceedings, (London), pp. 403-407, Institution of Electrical Engineers, 2000.

[4] R. Tabaksblat, R. J. Meier and B. J. Kip, "Confocal Raman microspectroscopy - theory and application to thin polymer samples", Appl. Spectrosc., vol.46, pp. 60-68, Jan. 1992.

[5] K. Uchida and N. Shimizu, "The effect of temperature and voltage on polymer chain scission in high-field region", IEEE Trans. Electr. Insul., vol.26, pp. 271-277, Apr. 1991.

[6] N. Shimizu, K. Uchida and S. Rasikawan, "Electrical tree and deteriorated region in polyethylene", IEEE Trans. Electr. Insul., vol.27, pp. 513-518, June 1992.

[7] C. Couprey, Analusis "Application of Raman microspectrometry to art objects", vol.28, pp. 39-46, Jan.-Feb. 2000.

[8] Z. Gao, I. Amaski, and M. Nakada, "A thermogravimetric study on thermal degradation of polyethylene", J. Anal. Appl. Pyrol., vol.67, pp. 1-9, Mar. 2003.

[9] T. Sugai, H. Yoshida, T. Shimada, T. Okazaki and H. Shinohara, "New synthesis of high-quality carbon nano-tubes by hightemperature pulsed arc discharge2, Nano Lets., vol.3, pp. 769773, June, 2003.

[10] A. S. Vaughan, S. J. Dodd and S. J. Sutton, "A Raman microprobe study of electrical treeing in polyethylene", submitted to J. Mater. Sci.

Author address: Alun S. Vaughan, ECS, Faraday Building, University of Southampton, Southampton SO17 1BJ, UK, Email: asv@ecs.soton.ac.uk 